\title{
Impression Materials and Storage Time Influence on Cast Models Accuracy
}

Fabiana Santos Gonçalves, Wallace de Freitas Oliveira, Luiza Anjos Soares, Hugo Lemes Carlo, Adérito Soares da Mota, Daniela Araújo Veloso Popoff, Carlos Jose Soares

\begin{abstract}
Background: There are a great amount of materials and techniques to use during dental prosthesis production that may confuse the dentist at the time of choice. Material's choice should be done considering ideal chemicophysical and biological properties and high detail reproduction.
\end{abstract}

Aims: The present study aimed to test the influence of differents impression materials and storage times on the dimensional accuracy of casting models.

Materials and methods: Four elastomeric materialspolysulfide, polyether, condensation silicone and addition silicone-were used to reproduce a master model of human teeth which were prepared for metaloceramic fixed prosthodontics. Six reference points were established on each tooth in order to determine four intervals: MD-mesial-distal, BL-buccal-lingual and CO-cervical-occlusal distance from premolar, and INTinterpillar distance-from the distal surface of the premolar to the mesial surface of the molar. The impressions were stored for half, 2 and 12 hours and pondered with a type IV gypsum.

Results: Cast measurements data were subjected to ANOVA $(4 \times 3)$, Tukey's $-\alpha=0.05$, Kruskal-Wallis and Dunn's tests $\alpha=0.05$. The interaction between materials and storage time presented statistically significant for VP and CO intervals. For each storage time, materials presented dimensional alteration statistically significant.

Conclusion: Impression materials accuracy was affected by the storage time. All materials presented different dimensional accuracy when evaluated in a same period of time. Polysulfide was the only material that presented stable behavior over the time.

Keywords: Dental impression materials, Elastomers, Physical properties.

How to cite this article: Gonçalves FS, de Freitas Oliveira W, Soares LA, Carlo HL, da Mota AS, Popoff DAV, Soares CJ. Impression Materials and Storage Time Influence on Cast Models Accuracy. Int J Experiment Dent Sci 2013;2(1):18-23.

Source of support: $\mathrm{Nil}$

Conflict of interest: None declared

\section{INTRODUCTION}

Modern operative dentistry uses a great quantity of impressions in many clinical applications. Elastomers were the first impression materials to present verified clinic results, being the first polysulfide use report described in the 50s. A that time, silicones were already used, but the polyether-based materials have only been introduced into clinical practice 10 years later. These new impression materials were able to reduce the working time and improve reproduction of detail. ${ }^{1,2}$

There are a great amount of materials and techniques to use during dental prosthesis production that may confuse the dentist when making the ideal choice. M aterial's choice should be done considering ideal chemicophysical and biological properties and high detail reproduction. Nevertheless, most of the impression materials generate imperfect molds and stone models, interfering on the qual ity of the final dental prosthesis. ${ }^{2-4}$

Some authors have verified that impression materials have, inevitably, some shrinkage level in the oral cavity due to the temperature variance of the external environment and to linear thermal expansion coefficient of these materials. Additionally, the material polymerization shrinkage promotes volumetric changes. This volumetric alteration is related with the tray influence, material adhesiveness level and polymer type. . $^{2,4-7}$

Stone models dimensional alterations can still be related to technical influence. ${ }^{1,5} \mathrm{~F}$ or putty/wash impressions, the taking procedures can be obtained by one-step or tw 0 -step techniques. In the first one the putty and wash materials are manipulated and simultaneously carried, in a custom tray, to the oral cavity, showing, according to some authors, worst dimensional accuracy. On the second technique, a preliminary putty impression is obtained, followed by a wash, resulting in a better accuracy. ${ }^{3,5}$

Unfortunately, stone models obtained by impression act show dimensional alterations. The materials compositions and the elapsed time between the impression taking and the model fabrication may influence the dimensional stability. ${ }^{1-8}$ Considering the great variety of techniques and materials compositions, the purpose of the present study was to determine the dimensional stability of four elastomeric impression materials considering the elapsed time between impression taking and stone model fabrication.

\section{MATERIALS AND METHODS}

Four elastomeric impression materials with different polymer composition were selected [polysulfide-PER (Permlastic, Kerr, M ichigan, EUA, lot: 4-234), polyetherIM (I mpregum, 3M/ESPE, Seefeld, Germany, lot: 0716600215), condensation silicone-ZE/OR (Zeta plus/ 
Oranwash, Zhermack, B adia Polesine, Italy, lot: 51044) and addition silicone-AD (A dsil, Vigodent, Rio de Janeiro, B razil, lot: 003/07)]. The dimensional stability was linearly measured in stone models obtained from impressions of a master model. The master model (Fig. 1) simulated a human superior arch, from third right molar to third left molar with acrylic resin mediated by two human teeth-one second premolar and one second molar (Research and Ethics Committee-Federal U niversity of U berlândia, \#177/07) and the absence of the first molar simulating prosthetic space. Resin teeth and natural teeth were fixed in a wax model (Clássico, A rtigos O dontológicos Clássico L tda, São Paulo, Brazil) and set parallel to each other in horizontal plan by means of a delineator (Delineador B2, Bioart, São Paulo, $B$ razil). The wax was then substituted by heat-polymerized acrylic resin (Clássico - incolor, A rtigos Odontológicos Clássico Ltda, São Paulo, Brazil). N atural teeth received preparations for metal ceramic crown restorations. Reference points were obtained on occlusal (Fig. 1) and vestibular sites (Fig. 2) of molar and premolar human teeth. The occlusal site received four orifices-mesial, distal, buccal and lingual and two on the buccal site-occlusal and cervical third. The orifices were made with carbide spherical drill

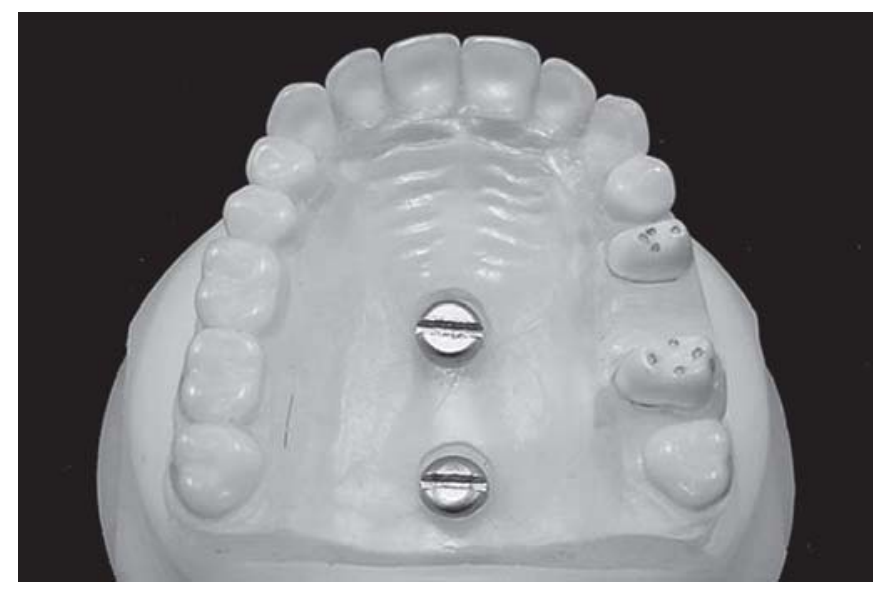

Fig. 1: Master model-visualization presenting occlusal orifices

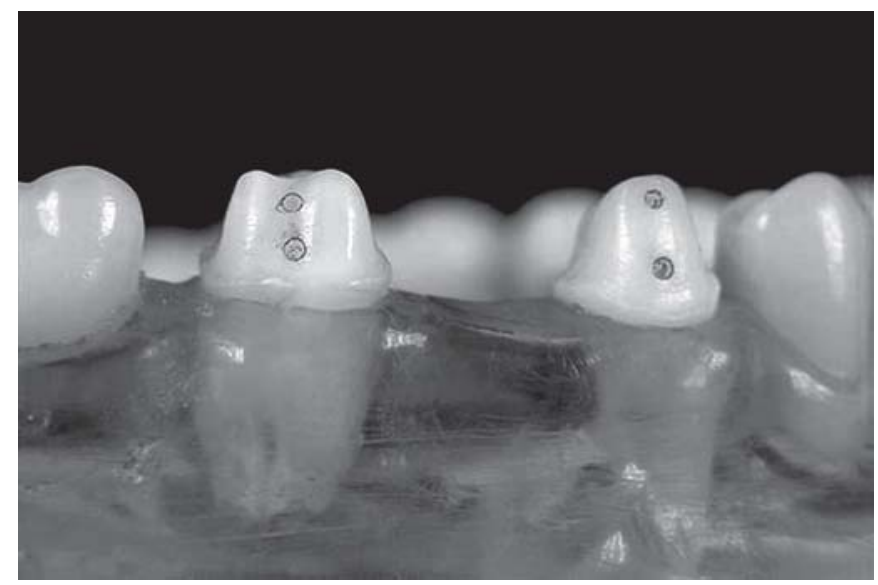

Fig. 2: Master model-visualization presenting vestibular orifices $\mathrm{n}^{1 / 4}$ (SS W hite, Rio de J aneiro, B razil), in a cavity preparation machine for the standardization of in vitro preparations. ${ }^{8}$ The internal margins of these orifices were used as references in order to determinate four measurement intervals: MD-mesial-distal, BL-buccal-lingual and COcervical-occlusal distance in the premolar tooth, and INTinterpillar distance from the distal surface of the premolar to the mesial surface of the molar tooth. B efore the impression taking act the master model was maintained in distilled water at $37^{\circ} \mathrm{C}$ during 15 minutes. ${ }^{7,9}$

The two-step putty/wash technique was used. All materials were manipulated according to manufacturers' recommendations. Perforated autopolymerized acrylic resin trays (V ipi Flash, Pirassununga, SP, B razil) with an internal relief of $3 \mathrm{~mm}$-to warrant wash material thickness standardization-w ere used. In order to minimize the acrylic resin shrinkage effect, trays were stocked in water during a minimum period of 24 hours before each impression. A copper-aluminum alloy spacer (D uracast M S, M arquart Cia L tda, São Paulo, Brazil) (Fig. 3)-3 mm thickness was positioned over teeth preparations before each putty impression, warranting wash material thickness standardization.

The impressions were maintained in $100 \%$ relative humidity and ambient temperature for 30 minutes, 2 and 12 hours. The storage time was defined simulating operational time of the dental office, in the following cases: (T1) cast fabrication 30 minutes after impression, granting material elastic recovery-still being considered as immediate; (T2) cast fabrication 2 hours after impression, simulating the process of sending the impression to the laboratory office; and (T3) cast fabrication 12 hours after impression, simulating the process of sending the impression to the laboratory office in cases which the impression is completed at the end of the day and cast at the morning of the next day. The stone model was fabricated using type IV gypsum (Herostone, V igodent, Rio

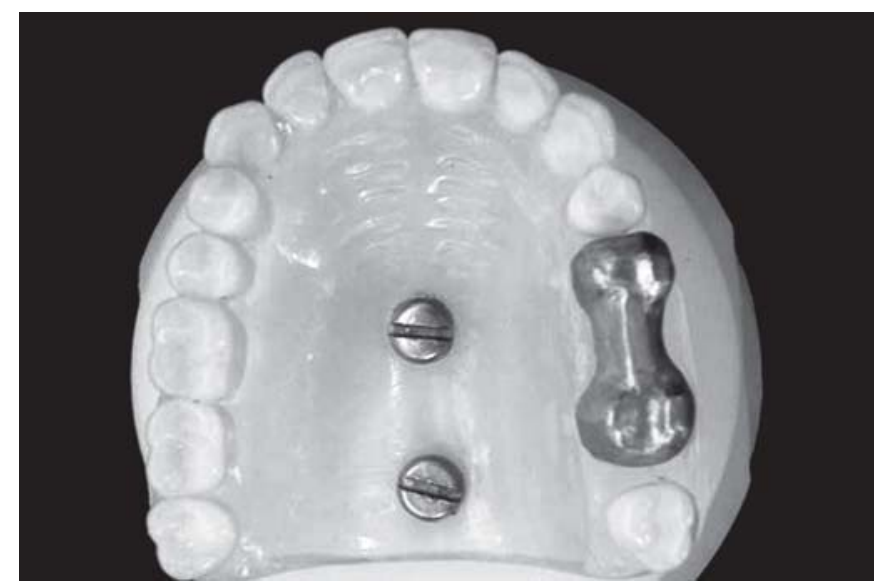

Fig. 3: Master model-metallic spacer positioned on master model 
de J aneiro, B razil, lot: 012-071) using manufacturer recommended water/pow der ratios and vacuum mixing (A 300, Polidental Ind. E Com. Ltda., São Paulo, Brazil). Impressions were poured using vibration and a slight angulation. Immediately after impressions being poured, a pyramidal support was positioned to permit model base standardization. U sing each of the impression materials, 120 cast models were randomly made $(n=10)$.

Samples (Fig. 4) were randomly evaluated by three independent examiners in a digital stereomicroscope (Olympus M easuring M icroscopeSTM , Orangeburg, USA), with $30 \times$ magnification and $0.0001 \mathrm{~mm}$ accuracy. Each examiner repeated three times each measure. Samples position on stereomicroscope was horizontally standardized using a flat base nylon apparatus with a pyramidal insertion for the stone die.

Dimensional accuracy was obtained subtracting stone die measurements from master model measurements. Data were subjected to A N OVA $(4 \times 3)$ and Tukey's $(\alpha=0.05)$ test for VP, CO and INT measurements. The MD

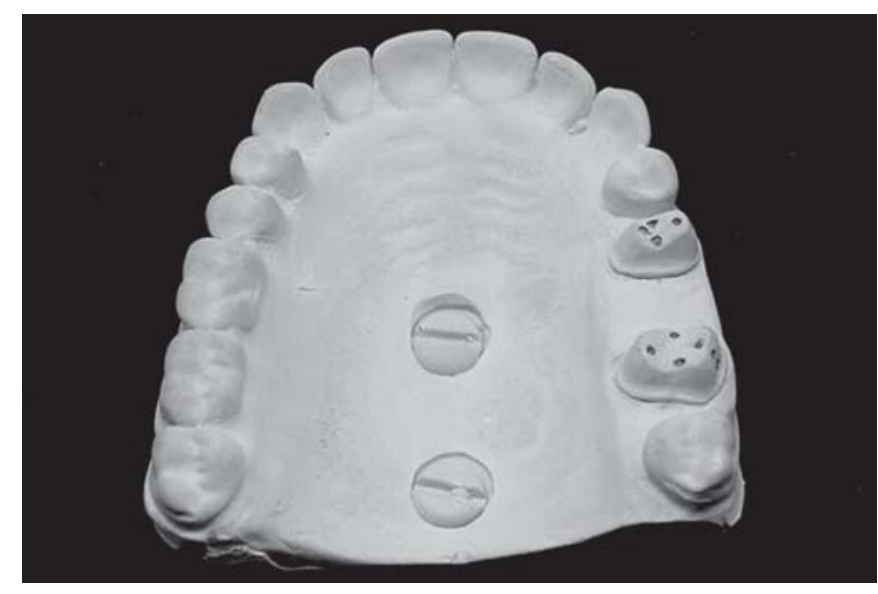

Fig. 4: Stone model

measurements were analyzed by K ruskal-W allis and Dunn's nonparametric tests $(\alpha=0.05)$.

\section{RESULTS}

Tables 1 to 4 show the mean values for VP, CO, INT and $M D$ measurements. Dimensional accuracy mean values for

\begin{tabular}{lccccc} 
& \multicolumn{2}{c}{ Table 1: Analysis of variance-MD interval } & & \\
Source & Sum of squares & $\mathrm{df}$ & Mean square & F & p-value \\
\hline Time & 0.002 & 2 & 0.001 & 1.097 & 0.338 \\
Material & 0.040 & 3 & 0.013 & 16.652 & 0.000 \\
Time $\times$ material & 0.008 & 6 & 0.001 & 1.680 & 0.133 \\
Error & 0.086 & 108 & 0.001 & & \\
\hline Total & 0.271 & 120 & & & \\
\hline
\end{tabular}

\begin{tabular}{lccccc} 
& \multicolumn{2}{c}{ Table 2: Analysis of variance-VP interval } & & \\
Source & Sum of squares & $\mathrm{df}$ & Mean square & F & p-value \\
\hline Time & 0.089 & 2 & 0.045 & 16.344 & 0.000 \\
Material & 0.199 & 3 & 0.066 & 24.321 & 0.000 \\
Time $\times$ material & 0.081 & 6 & 0.014 & 4.954 & 0.000 \\
Error & 0.295 & 108 & 0.003 & & \\
\hline Total & 0.786 & 120 & & & \\
\hline
\end{tabular}

\begin{tabular}{|c|c|c|c|c|c|}
\hline Source & Sum of squares & $d f$ & Mean square & $\mathrm{F}$ & $p$-value \\
\hline Time & 0.004 & 2 & 0.002 & 2.259 & 0.109 \\
\hline Material & 0.058 & 3 & 0.019 & 20.417 & 0.000 \\
\hline Time $\times$ material & 0.034 & 6 & 0.006 & 5.924 & 0.000 \\
\hline Error & 0.102 & 108 & 0.001 & & \\
\hline Total & 0.216 & 120 & & & \\
\hline
\end{tabular}

\begin{tabular}{|c|c|c|c|c|c|}
\hline Source & Sum of squares & $\mathrm{df}$ & Mean square & F & n-value \\
\hline Time & 1.937 & 2 & 0.968 & 1.022 & 0.363 \\
\hline Material & 2.973 & 3 & 0.991 & 1.046 & 0.375 \\
\hline Time $\times$ material & 5.833 & 6 & 0.972 & 1.026 & 0.412 \\
\hline Error & 102.301 & 108 & 0.947 & & \\
\hline Total & 117.579 & 120 & & & \\
\hline
\end{tabular}


Impression Materials and Storage Time Influence on Cast Models Accuracy

\begin{tabular}{|c|c|c|c|c|c|c|c|}
\hline \multirow[t]{2}{*}{ Material } & \multirow{2}{*}{$\frac{\text { Interval MD }}{\text { Time* }}$} & \multicolumn{3}{|c|}{ Interval VP } & \multicolumn{3}{|c|}{ Interval CO } \\
\hline & & 30 mins & $2 \mathrm{hrs}$ & $12 \mathrm{hrs}$ & 30 mins & $2 \mathrm{hrs}$ & $12 \mathrm{hrs}$ \\
\hline Polysulfide & $0.030^{b}$ & $-0.039^{c, A}$ & $0.029^{b, A}$ & $0.016^{\mathrm{b}, \mathrm{A}}$ & $0.031^{a, A}$ & $0.041^{a, A}$ & $0.047^{a, A}$ \\
\hline Condensation silicone & $0.062^{\mathrm{a}}$ & $0.014^{b c, B}$ & $0.013^{b, B}$ & $0.113^{\mathrm{a}, \mathrm{A}}$ & $0.026^{b, B}$ & $0.015^{a, A}$ & $0.034^{a, A}$ \\
\hline Polyether & $0.032^{\mathrm{ab}}$ & $0.048^{a b, B}$ & $0.065^{\mathrm{a}, \mathrm{AB}}$ & $0.144^{\mathrm{a}, \mathrm{A}}$ & $0.005^{\mathrm{ab}, \mathrm{B}}$ & $0.022^{a, A B}$ & $0.038^{a, A}$ \\
\hline Addition silicone & $0.010^{c}$ & $0.061^{a, A}$ & $0.020^{\mathrm{ab}, \mathrm{A}}$ & $0.040^{\mathrm{b}, \mathrm{A}}$ & $0.006^{a b, A}$ & $0.025^{b, B}$ & $0.044^{b, B}$ \\
\hline
\end{tabular}

Media followed by distinct letters differ among themselves by Tukey test $(p<0.05)$; Lower case letters: Comparison among materials; Capital letters: Comparison among each material over the time; *For interval, MD did not have significant alteration according to the time

premolar, considering materials and storage time, are demonstrated on Table 5. The interpillar mean value measurements, according to materials and storage time are on Table 6. Negative and positive values correspond to bigger or smaller casts than the master model, respectively.

$M$ aterial behavior as a function of time had meaning presented statistical difference depending on the analyzed measurement interval. For the M D measurement there was only statistical difference concerning to material type $(p=0.000)$.

Addition silicone presented the best values of dimensional accuracy, followed by polysulfide, polyether and condensation silicone. The VP measurement analysis presented that IM and ZE/OR showed an increase of the size of the model along time and PER and AD showed stability. The $\mathrm{CO}$ measurement presented an increase of the size of the model along time for the materials IM and ZE/OR. AD material presented reduction of the samples size and PER dimensional stability. Interpillar measurement presented no statistically significant difference related to material $(p=0.375)$, storage time $(p=0.363)$ and factors interaction $(p=0.412)$.

\section{DISCUSSION}

The hypothesis tested that composition and storage time would influence material dimensional stability was acceptable. Just polysulfide impressions presented stable

\begin{tabular}{|c|c|}
\hline \multirow[t]{2}{*}{ Material } & Interval INT \\
\hline & Time* \\
\hline Polysulfide & $0.061^{\mathrm{a}}$ \\
\hline Condensation silicone & $0.119^{a}$ \\
\hline Polyether & $0.135^{\mathrm{a}}$ \\
\hline Addition silicone & $0.115^{a}$ \\
\hline
\end{tabular}

Media followed by distinct letters differ among themselves by Tukey test $(p<0.05)$; *F or interval, INT did not have significant alteration according to the time behavior over time, with no statistical significant difference on three storage periods and four analyzed dimensions. The other groups presented dimensional alteration at least in one of the measured intervals. Besides, these samples presented more expansion results (positive values) than contraction results (negative values).

Elastomeric materials are susceptible to permanent deformation, which can justify the nonuniformity achieved in this experiment. The surface of natural teeth presents great adherence to the impression material and also the reference points in orifice shape presents some retentiveness, causing difficulty on the impression discharge or deformation on it. This deformation is related to material's incapacity of totally returning to its original dimension. ${ }^{1,7,10}$

Polysulfide material polymerizes by increasing the extent of the polymeric chains and by cross-linking of the $\mathrm{SH}$ groups in its basic molecule in the presence of lead dioxide. This reaction occurs by condensation process and releases water as byproduct. Water evaporation on impression surface results in distortion, affecting the dimensional stability of the material after setting. The results observed in this material showed no dimensional al teration over time when compared to master model. Probably the storage medium with $100 \%$ of relative humidity (water saturated) helped the impression to maintain dimensional stability by reducing water liberation of the sample to the environment. All condensation silicone impressions presented different measures when compared to master model. Probably it occurred because of the volatilization of the alcohol formed as byproduct. Alcohol evaporation results in distortion of the impression, affecting its dimensional stability. ${ }^{1,11}$

Polyether polymer-based material polymerizes by means of a reaction between aziridine rings, situated at the end of the chains, of each polyether molecule, in the presence of sulfonated ester. In this cure reaction there is no byproduct development, which helps dimensional stability of the impression. However, unlike other materials, polyether is highly hydrophilic, which may lead to water absorption from 
the atmosphere or the storage medium. ${ }^{1,11-13}$ The material can also absorb water from the gypsum, causing expansion of the mould and therefore smaller models. Hence, it would be expected water sorption to cause changes in the samples decreasing the distances measured. ${ }^{4,5,7-9} \mathrm{H}$ owever, changes were observed increasing both horizontal (V P) and vertical (CO) measures, which suggests that the material did not presented a hydrophilic aspect. ${ }^{11}$

Addition silicone polymerization occurs without the release of by product, showing no dimensional change over time than the others and it is therefore considered dimensional stable by most studies. Significant changes were observed in the models obtained from addition silicone only to $\mathrm{CO}$ measurement in the storage time of 2 and 12 hours, being the casts smallers than the master model. The casts probably were smaller due to the vertical component of the polymerization shrinkage toward the occlusal portion when the impression is tightly attached to the tray. N evertheless, the accuracy and stability of this material were maintained for most of the distances measured. ${ }^{1,12}$

According to the results of this study it is observed that the dimensional changes that occurred between 30 minutes and 12 hours of storage are significant and dependent on the material used. Overall, the results indicate that the cast models for polyether, condensation silicone and addition silicone must be made within a shorter time (30 minutes) in order to prevent dimensional changes between impression and model. This relationship material/casting time differs from the instructions of the manufacturers that indicate 30 minutes and 14 days for $I M, 30$ minutes and 3 days for the ZE/OR, and 30 minutes and 7 days for AD. Polysulfide, if properly packaged in an environment with $100 \%$ relative humidity, produced results which showed that their cast may be casted up to 12 hours after impression taking. This relationship material/casting time is in accordance with the instruction of the manufacturer that recommends 30 minutes to 8 hours.

This experiment tried to access laboratory results correlating to situations presented by the clinical reality. Although considering the teeth surface reproduced and the conditions of humidity and temperature presented by oral cavity it is recognized that not always it is possible to simulate all the factors to which materials are subjected during clinical use. Similar experiments should be conducted considering three-dimensional or volumetric assessments to guarantee better understanding of the dimensional behavior of the materials over time.

Within the limitations of this in vitro study and in accordance with the results obtained, it is possible to conclude that condensation silicone, addition silicone and polyether-based materials presented dimensional changes as a function of time in at least one of the dimensions examined, when compared to the master model; polysulfidebased material presented dimensional stability up to 12 hours after impression technique; the cast models showed behavior of expansion or contraction, being the first more common; all materials showed different measures when evaluated at the same period of time.

\section{REFERENCES}

1. D onovan TE, Chee WW L. A review of contemporary impression materials and techniques. Dent Clin N A m 2004;48:445-70.

2. Papadogiannis $D, L$ akes $R$, Palaghias $G$. Effect of storage time on the viscoelastic properties of elastomeric impression materials. J Prosthodont Res 2012;56:11-18.

3. Nassar U, Aziz T, Flores-M ir T. Dimensional stability of irreversible hydrocolloid impression materials as a function of pouring time: A systematic review. J Prosthet Dent 2011;106:126-33.

4. K anehira M, Finger WJ, Endo T. V olatilization of components from and water absorption of polyether impressions. J Dent 2006;34:134-38.

5. A I-Barki IA, Hussey D, AI-Omari WM. The dimensional accuracy of four impression techniques with the use of additiontype silicone impression materials. J Clin Dent 2007;18:29-33.

6. Hiraguchi $H, K$ aketani $M$, Hirose $H, Y$ oneyama $T$. Effect of immersion disinfection of alginate impressions in sodium hypochlorite solution on the dimensional changes of stone models. Dent M ater J 2012;31:280-86.

7. Endo T, Finger WJ. Dimensional accuracy of a new polyether impression material. Quintessence Int 2006;37:47-51.

8. Soares CJ, M artins LR, Pfeifer J M, Gianini M. Fracture resistance of teeth restored with indirect composite and ceramic M OD systems. Quintessence Int 2004; 35:281-86.

9. Thongthammachat S, M oore BK, B arco M T, Hovijitra S, B rown DT, A ndrés CJ . Dimensional accuracy of dental casts: Influence of tray material, impression material and time. J Prosthodont 2002;11:98-108.

10. B alkenhol M, H aunschild S, Erbe C, W östmann B. Influence of prolonged setting time on permanent deformation of el astomeric impression materials. J Prosthet Dent 2010;103:288-94.

11. A nusavice KJ. Phillips' science of dental materials (11th ed). St. L ouis: Elsevier 2003:212-31.

12. Vasconcellos FEC, A ndreullo RF, Sabrosa CE, N eisser M P, R ego M A. Dimensional stability of casts obtained with polyether and addition reaction silicone after disinfection with sodium hypochlorite and peracetic acid. Rev Bras Odontol 2012;1: 55-60.

13. Rodriguez JM, Bartlett DW. The dimensional stability of impression materials and its effect on in vitro tooth wear study. Dent $M$ ater 2011;27:253-58.

\section{ABOUT THE AUTHORS}

\section{Fabiana Santos Gonçalves}

Postgraduate Student, Department of D entistry, Federal University of M inas Gerais, M inas Gerais, B razil 


\section{Wallace de Freitas Oliveira}

Graduate Student, D epartment of D entistry, State U niversity of M ontes Claros, M G, Brazil

\section{Luiza Anjos Soares}

Graduate Student, Department of Dentistry, Faculdades U nidas do Norte de M inas, M G, B razil

\section{Hugo Lemes Carlo}

Professor, Department of Dentistry, Federal University of Paraíba Paraíba, Brazil

\section{Adérito Soares da Mota}

Professor, D epartment of D entistry, Federal U niversity of U berlândia M inas Gerais, B razil

\section{Daniela Araújo Veloso Popoff (Corresponding Author)}

Professor, D epartment of D entistry, State U niversity of M ontes Claros M inas Gerais, B razil, e-mail: danielavelloso@yahoo.com.br

\section{Carlos Jose Soares}

Professor, D epartment of D entistry, Federal U niversity of U berlândia M inas Gerais, B razil 